\title{
Horizontal projections of area 17 in Cebus monkeys: metric features, and modular and laminar distribution
}

A.K.J. Amorim and C.W. Picanço-Diniz
Departamento de Fisiologia, Universidade Federal do Pará, Belém, PA, Brasil

\begin{abstract}
Correspondence
A.K.J. Amorim

R. São Salvador, 30/310

Bairro Flamengo

22231-130 Rio de Janeiro, RJ

Brasil

Received January 30, 1997

Accepted September 30, 1997

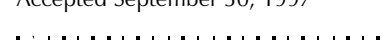

Key words

- V1 intracortical projections

- Neurons

- Axon terminals

- Visual cortex

- Primate

\section{Introduction}

Intrinsic connections in the primate visual cortex have been the subject of a number of recent studies on several visual areas (1-10). For reviews see also Lund et al. (11) and Casagrande and Kaas (12). These results suggest different cell morphologies, laminar origins, targets and degrees of modular segregation for intrinsic connections of the primate brain encouraging further investigation on the morphofunctional organization of those connections. Horizontal connections of particular interest to the present study have shown different degrees of segregation linking different cortical compartments $(1,3,5,7,11,13-15)$. Studies using high resolution neurotracers such as peroxidase or biocytin in New World primates $(2,4,14,16)$ emphasize the degree of modular segregation for intrinsic connections. However, so far there are no studies correlating metric features of intrinsic axon terminals with their laminar or modular distribution in V1 of New World primates. Thus, the present study is the first detailed description of the intrinsic projections of V1 of Cebus apella attempting to address two questions, i.e., the morphometric features of the intrinsic projections as well as the modular and laminar distributions of the neuronal processes.

A partial description of some of these results has been presented elsewhere (17).

\section{Material and Methods}

Five adult male Cebus apella were anesthetized by intramuscular injection of a mixture of ketamine $(11 \mathrm{mg} / \mathrm{kg})$ and xylazine 
$(0.55 \mathrm{mg} / \mathrm{kg})$ prior to surgery. Supplementary doses were given as needed. Five to 15 iontophoretic injections of $5 \%$ biocytin diluted in $0.1 \mathrm{M}$ Tris/0.2 M KCl, $\mathrm{pH} 7.9$, were made into the operculum of each hemisphere of two subjects. A 7-10- $\mu$ A DC negative current was applied through pipette tips 20$50 \mu \mathrm{m}$ in diameter ( $7 \mathrm{~s}$ on, $7 \mathrm{~s}$ off) to deliver the tracer for a period of 10-20 min. Three other animals received implants of saturated biocytin pellets in the operculum (18). Double or triple labeling with cytochrome oxidase (CYTOX) and/or NADPH-diaphorase (NADPH-d) and biocytin was perfomed on the same sections. Histochemical reactions for metabolic markers were carried out before the biocytin reaction according to the protocols of Wong-Riley (19) for CYTOX and of Scherer-Singler et al. (20) for NADPH-d. Before the histochemical reaction for the visualization of biocytin-labeled terminals, sections were incubated in avidin-biotin complex (ABC) followed by histochemistry for horseradish peroxidase (HRP) using the glucose oxidase-diaminobenzidine (DAB)-nickel protocol of Shu et al. (21). After processing, the sections were mounted on gelatinized slides, dehydrated, cleared and coverslipped with DPX (a mixture of distyrene, tricresyl phosphate and xylene). In order to identify cortical lamination some sections were counterstained with cresyl violet.

For 3-D computer reconstruction each process was digitized directly from the slide using a 60X oil immersion objective (Nikon) on a Nikon microscope with a motor-driven Z-encoder which allowed the $\mathrm{x}, \mathrm{y}$ and $\mathrm{z}$ coordinates of the points entered to be stored in a computer (Silicon Graphics Personal Iris, Mountain View, CA). For the purposes of the present investigation we only digitized processes that were included in just one 150$200-\mu \mathrm{m}$ thick tangential or parasagittal section; they do not necessarily correspond to a complete reconstruction of the whole axonal or dendritic tree. For 3-D reconstruction we only sampled axon terminals and dendritic trees that displayed true ends along the section. Smaller trees showing thicker cut ends were not included in the sample. All software for data entry and data analysis was developed in the Neurobiology Research Center Imaging Facility (Birmingham, AL, USA) by J. Gemmill. We measured a number of metric features including densities of branching points, branches and boutons, combined with segment length analysis and branching angles. To assess the average density of branching points, branches or boutons, we simply divided the total number of appendages by the total length of the dendrite or axon terminal. The total length of the dendritic trees or axon terminals was obtained by adding the length of all segments. At all branching points we measured the angle (in degrees) between each pair of dendritic or axon segments. Since shrinkage in the $\mathrm{z}$ axis would impose a distortion of the angle between branches the digitized images of neurons were "stretched" by the degree of tissue shrinkage assuming the shrinkage process to be linear. To compute the angle between each pair of branches of the same parental segment we assumed that 3 sampling points away from the branching points were linked by a straight line. The angle between these straight lines was then measured. The means were calculated for dendrites and for each group of axon terminals and the $t$-test $(\mathrm{P}<0.05)$ was applied in each case to evaluate the statistical significance of the differences.

\section{Results}

\section{Cortical layers and blobs}

Figure 1A illustrates a parasagittal section reacted for NADPH-d (A) and counterstained with Nissl (B) to visualize the cortical layers. Layer numbers refer to Hässler's (22) nomenclature modified by Lachica et al. (4). Whenever necessary Brodmann's nomenclature(23) will be used and cited in parentheses along the 
A

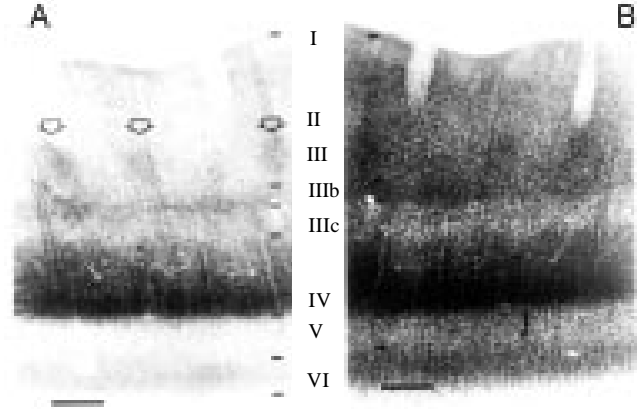

Figure 1 - Parasagittal section reacted for NADPH-d (A) and counterstained with Nissl (B) to visualize the cortical layers. Layer numbers according to Hässler's (22) nomenclature modified by Lachica et al. (4). Open arrows point to blobs. Scale bar $200=\mu \mathrm{m}$.



Figure 2 - Low power photomicrograph of a tangential section through $\mathrm{V} 1$ double labeled for biocytin and cytochrome oxidase (CYTOX) illustrating a biocytin iontophoretic injection site (open arrow) relative to the CYTOX blobs. Filled arrows point to a row of blobs and arrowheads point to individual blobs. Scale bar $=400$ $\mu \mathrm{m}$.

text and in the figures. Blobs are indicated by arrows in layer III (Figure 1A). Note that layer IV (IVc according to Brodmann's nomenclature) shows at least three distinct sublaminae with the lower third portion of the lamina being much darker than the more superficial two thirds. However, the upper two thirds can also be subdivided into sublaminae: the most superficial and thickest portion of layer IV showed poor NADPH-d activity. Layers V and VI are distinguished from each other by the more intense reactivity of layer VI for NADPH-d histochemistry (see also Figure 5A). This pattern of reactivity for NADPH-d for V1 of Cebus apella, with clear modular and laminar organizations illustrated in Fig-
$\mathbf{A}$
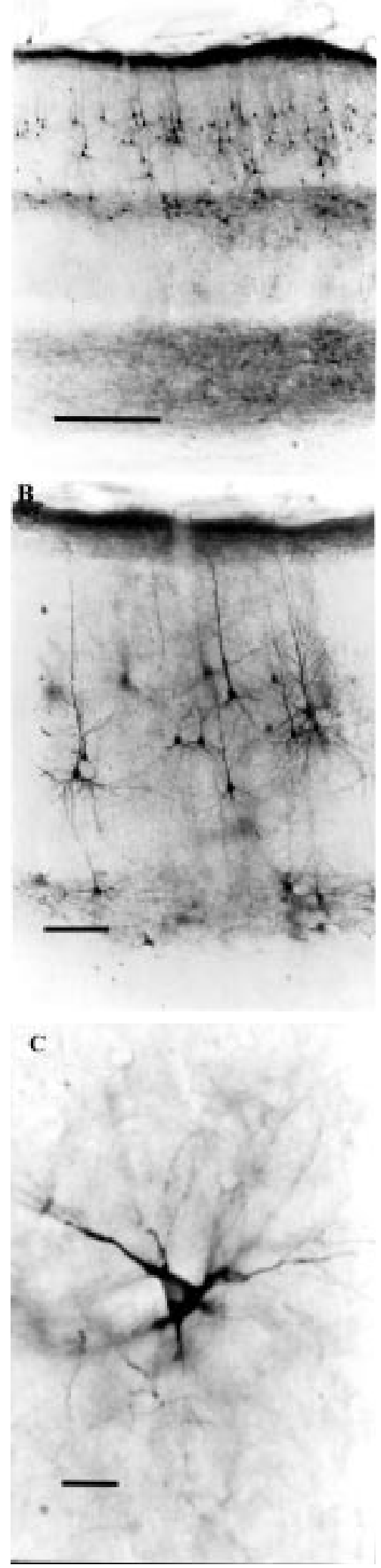

Figure 3 - Photomicrographs of retrogradely labeled cells of $\mathrm{V}_{1}$ after implanting biocytin gels. $A$, Low power photomicrograph illustrating two distinct rows of labeled neurons in the superficial and deep portions of layer III. Scale bar $=400 \mu \mathrm{m}$. B, High power photomicrograph showing different size pyramidal cells in the superficial sublayers (IIla and IIlb). Scale bar $=100 \mu \mathrm{m}$. $C$, Typical stellate cell from layer IIIc (IVb). Scale bar $=20 \mu \mathrm{m}$. 
A

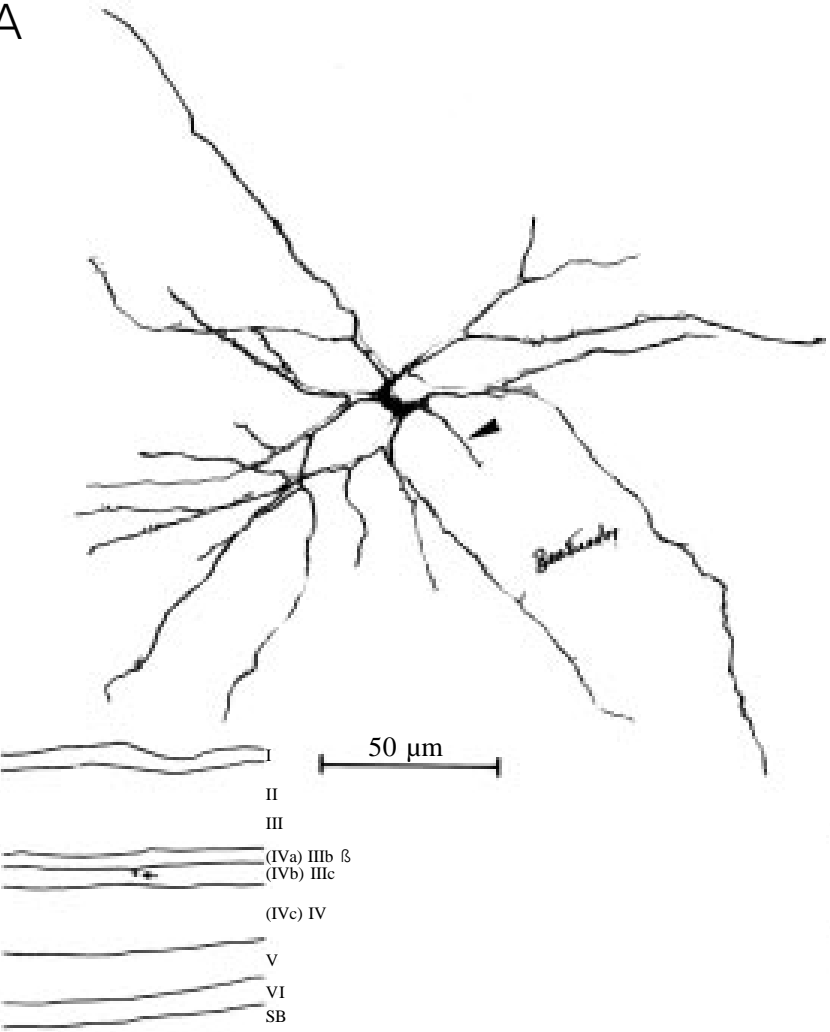

C

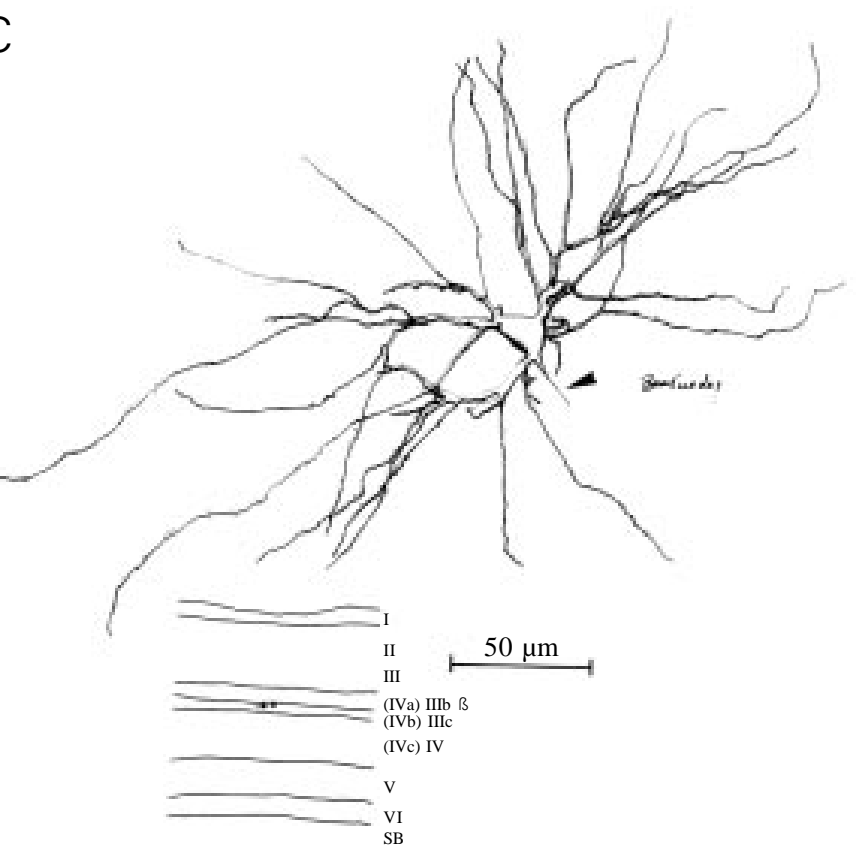

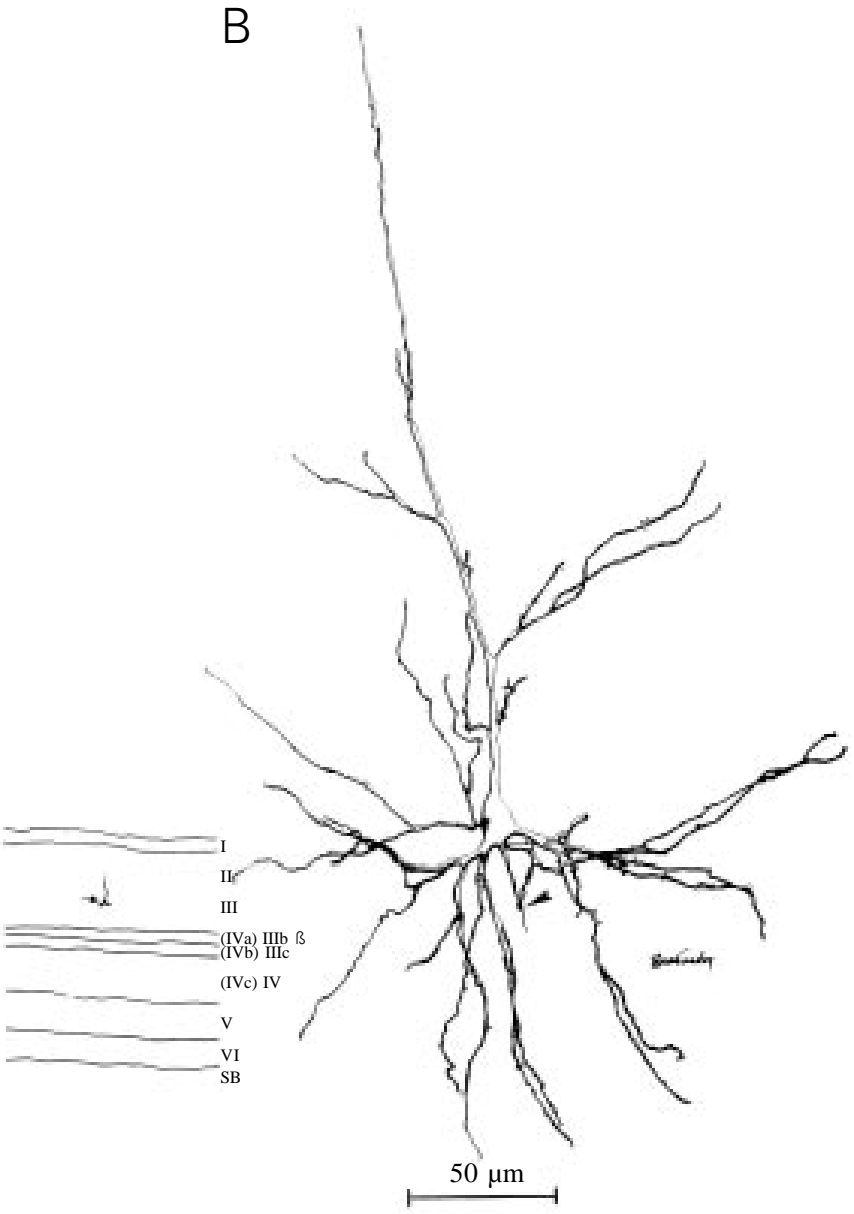

Figure 4 - Camera lucida drawings of retrogradely labeled cells of V1 after implanting biocytin gels. $A$, Star pyramidal; $B$, pyramidal; $C$, stellate neurons. Arrowheads point to axons. Insets illustrate the location of these cells relative to the cortical layers. SB, white matter. 
ure 1 , is similar to that previously described by Rosa et al. (24) using CYTOX histochemistry. The borders of the major subdivisions between cortical layers, as revealed by NADPH-d histochemistry in the present study, are virtually the same as obtained by Nissl staining and they were more evident in a double-labeled section (Figure 1B). Figure 2 is a low power photomicrograph of a tangential section of V1 to illustrate the size of a biocytin iontophoretic injection site (open arrow) in relation to the blobs of cytochrome oxidase, after double labeling. We observed well-individualized blobs (arrowheads) or rows of confluent blobs (small filled arrows) forming beaded strips.

\section{Cell morphology and laminar distribution}

Figure 3 illustrates retrogradely labeled cells of V1 after implantation of biocytin gels. At low power (Figure 3A) at least two distinct horizontal rows of labeled neurons can be seen in the superficial (layers IIIa and IIIb) and deep portions of layer III (layer IIIc). At high power (Figure 3B) pyramidal cells of different sizes can be seen in the superficial layers (IIIa and IIIb) with long apical dendrites and basal lateral dendrite tufts. Figure 3C illustrates a typical stellate cell from layer IIIc (IVb) showing the stellate pattern of dendritic branching and no apical dendrite. Similar results were obtained after the iontophoretic injections, except for the fact that in these cases retrograde transport was much less significant, precluding the possibility of clearly identifying neuron morphology. Figure 4A,B,C illustrates camera lucida drawings of pyramidal and stellate neurons. A particular type of pyramidal cell, the star pyramid of layer IIIc (IVb), is shown in A; B and $C$ represent pyramidal and stellate cells, respectively.

Thus, three different cell morphologies were found after retrograde transport of biocytin: pyramids, star pyramids and stel-

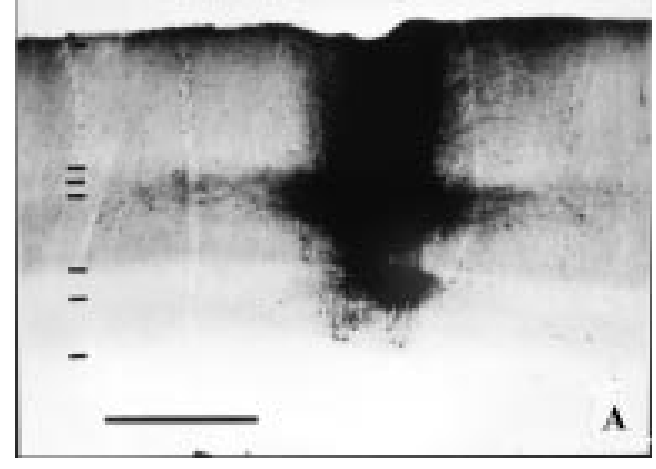

Figure 5 - $A$, Low power photomicrograph illustrating anterograde and retrograde transport near the injection site. Scale bar $=500 \mu \mathrm{m}$. B, High power photomicrograph illustrating in detail a pyramidal cell in infragranular layers. Thick arrows point to an axon. Thin arrow points to spines on dendrite. Scale bar $=10 \mu \mathrm{m}$.

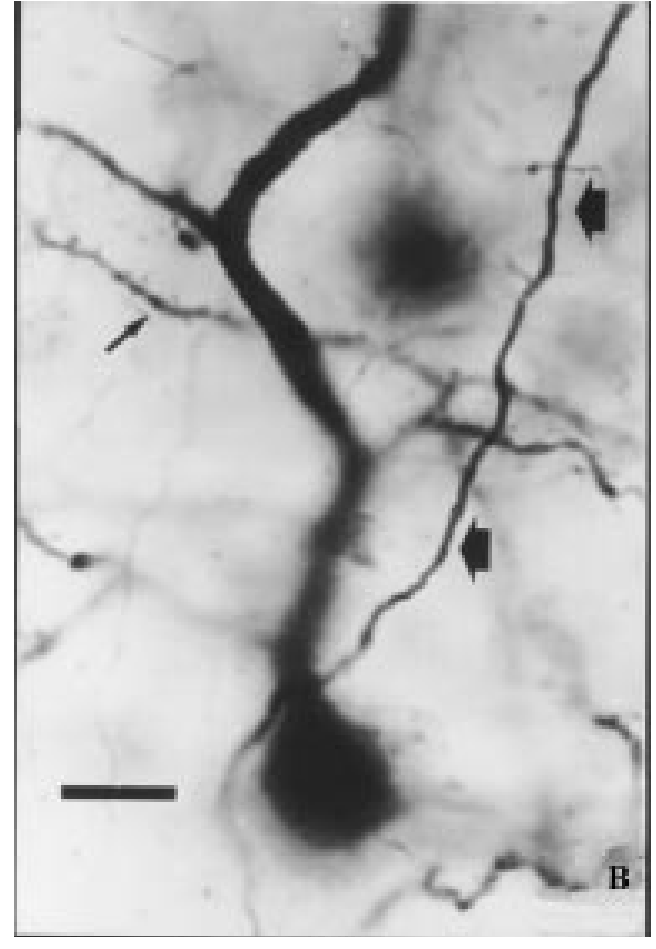

late cells. Pyramidal cells were mainly but not exclusively located in supragranular layers, predominantly in layers IIIa and IIIb, with highest density being observed in IIIa. Stellate cells and star pyramids were located particularly in layer IIIc (IVb). In register with the injection site (Figure 5A,B), we also found pyramidal cells in infragranular layers. However, no retrograde labeling was observed beyond the limits of the injection site in infragranular layers (Figure 5A). 
Figure 6 - Camera lucida drawing (top) and 3-D reconstructions (middle and bottom) of axon terminals in V1. Top, Type la axon; middle, type lb axon; bottom, type $\mathrm{Il}$ axon. Scale bar $=100 \mu \mathrm{m}$.

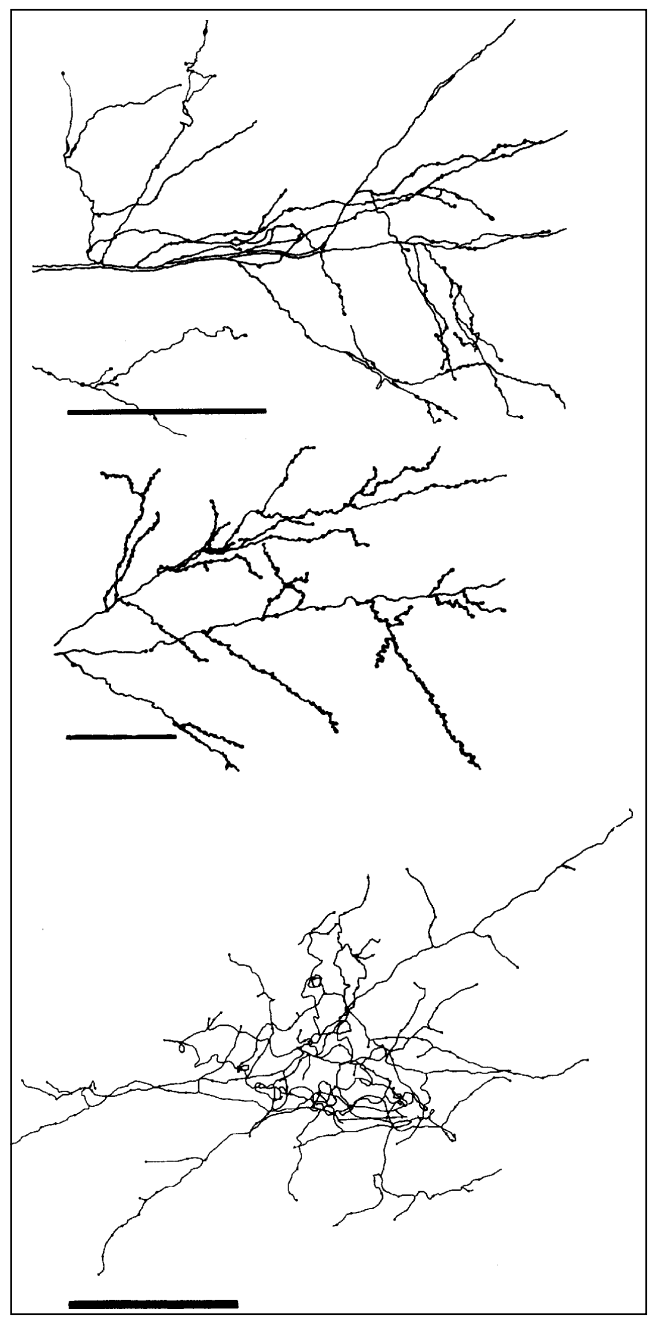

Morphology and modular and laminar
distributions of intrinsic axon terminals

All terminals analyzed were located in supragranular layers and resulted from injection sites restricted to granular or supragranular layers (Figure 5A). The largest injection sites correspond to $500 \times 800 \mu \mathrm{m}$ in the horizontal and vertical axes, respectively, as measured in parasagittal sections. Reconstructed axon terminals correspond to a fraction of the entire axonal tree. Comparison of some morphological features such as tree appearance, branching pattern, and bouton size and shape led us to distinguish two groups of axon terminals in V1 of Cebus apella that were named type I and II (Figure
6). Each axonal tree displayed principal (thicker), intermediate and terminal (thinner) segments. The principal segment was identified nearest the injection site, isolated from the other branches. In type I axon terminals this segment ran larger distances than type II terminals before giving rise to new branches. Two groups of type I axon terminals were distinguished: type Ia (Figure 6, top) showed a number of intermediate and terminal branches that organized themselves, forming small patches interconnected by long segments not covered with cell appendages; type Ib (Figure 6, middle) axon terminals, in contrast to type Ia, did not form patches, but gave rise to a number of intermediate branches along the principal segment. The principal segment became thinner as it diverged to give rise to new distal branches. Type II axon terminals (Figure 6, bottom) displayed a rather compact and complex arborization, with almost no variation in the thickness of their branches, rendering it very difficult to identify the principal segment. They showed very short and thick intermediate segments branching in various directions. Figure 7 shows typical "en passant" and "terminaux" boutons for different axon terminals. Table 1 summarizes the qualitative characteristics of these groups.

\section{Laminar distribution}

Iontophoretic injections including layers I to IV in the operculum of V1 were used to study the laminar distribution of intrinsic axon terminals (Figure 5A). The results are summarized in Tables 2 and 3 . Using the criteria previously described for sampling axon terminals for reconstruction, $76 \%$ of all cases could be classified as belonging to group I. The identification of the principal segment of type II axon terminals was not as clear as for type I axon terminals.

All type I axon terminals were found in the supragranular layers, especially in layers 


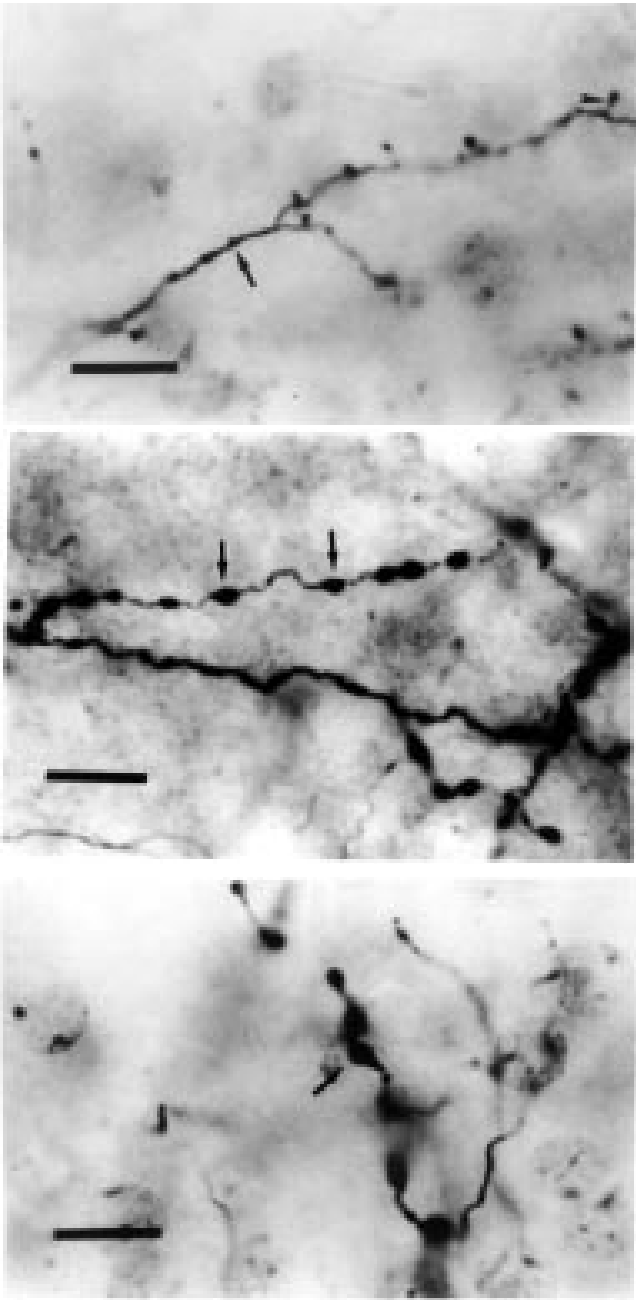

Figure 7 - High power photomicrographs illustrating "en passant" and "terminaux" boutons for different axon terminals. Top, Type la axon; middle, type lb axon; bottom, type II axon. Arrows point to "en passant" boutons. Arrowhead points to "terminaux" bouton. Scale bars $=100 \mu \mathrm{m}$.

III (IIIa, IIIb $\alpha$ (IIIb), IIIb $\beta$ (IVa) and IIIc $(\mathrm{IVb}))$. Some type I terminals had their primary branches in layers I, II and III (AXI4, AXI5, AXI7; Table 2) and tree in the same layers. Most of type I axon terminals had their primary segment restricted to a single layer, running along varying distances before giving rise to end branches that, in general, were not restricted to a single layer. This fact may indicate that these axons could participate in interlaminar circuits within one cortical area. In the case of type II axon
Table 1 - Qualitative analysis of intrinsic axon terminals of V1 of Cebus apella.

\begin{tabular}{lccccc}
\hline \multirow{2}{*}{$\begin{array}{l}\text { Axon } \\
\text { terminal }\end{array}$} & Patches & & \multicolumn{2}{c}{ Boutons } & Branching \\
\cline { 3 - 5 } & & & "En passant" & "Terminaux" & \\
\hline la & + & + & + & sparse \\
lb & - & + & - & sparse \\
II & - & + & - & dense
\end{tabular}

Table 2 - Laminar distribution of type I axon terminals of V1 of Cebus apella.

\begin{tabular}{|c|c|c|}
\hline $\begin{array}{l}\text { Axon } \\
\text { terminals }\end{array}$ & Principal segment & End segments \\
\hline AXI1 & $\mathrm{IIIb} ß$ & 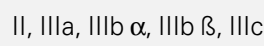 \\
\hline AXI3 & $\mathrm{III} / \mathrm{IV} \alpha$ & II, III \\
\hline AXI4 & $\|/\| \|$ & I, II \\
\hline AXI5 & $\|/\| \|$ & I, II, III \\
\hline AXI7 & I & I, II \\
\hline AXI12 & IIIC & IIIa, IIIb, IIIc \\
\hline AXI13 & $\mathrm{II} \mathrm{lb} ß / \mathrm{II} \mathrm{c}$ & \|\|$b \alpha,\|l b \beta\| l c$, \\
\hline
\end{tabular}

Table 3 - Laminar distribution of supragranular type II axon terminals of V1 of Cebus apella.

\begin{tabular}{|c|c|}
\hline Terminals & Layers \\
\hline AXII6 & IIIb ß, IIlc \\
\hline AXII9 & 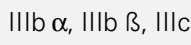 \\
\hline AXII10 & IIIc, IV $\alpha$ \\
\hline AXII11 & IIIb ß, IIIc \\
\hline
\end{tabular}

terminals, terminal arborizations did not exceed the limits of one or two sublayers. Thus, it is possible that type II terminals play a role in regulating both inter- and intralaminar activity through possible synaptic contacts with neurons located in the same layer.

\section{Modular distribution}

Figure 8 and Table 4 illustrate the different degrees of segregation as well as the spatial distribution of axon terminal arboriza- 
Figure 8-3-D reconstructions of axon terminals in relation to the modular organization of $\mathrm{V} 1$. A, Complete segregation inside blobs. Scale bar $=400 \mu \mathrm{m}$. $B$, Complete segregation outside blobs. C, Incomplete segregation. Scale bars in $B$ and $C=100$ $\mu \mathrm{m}$.

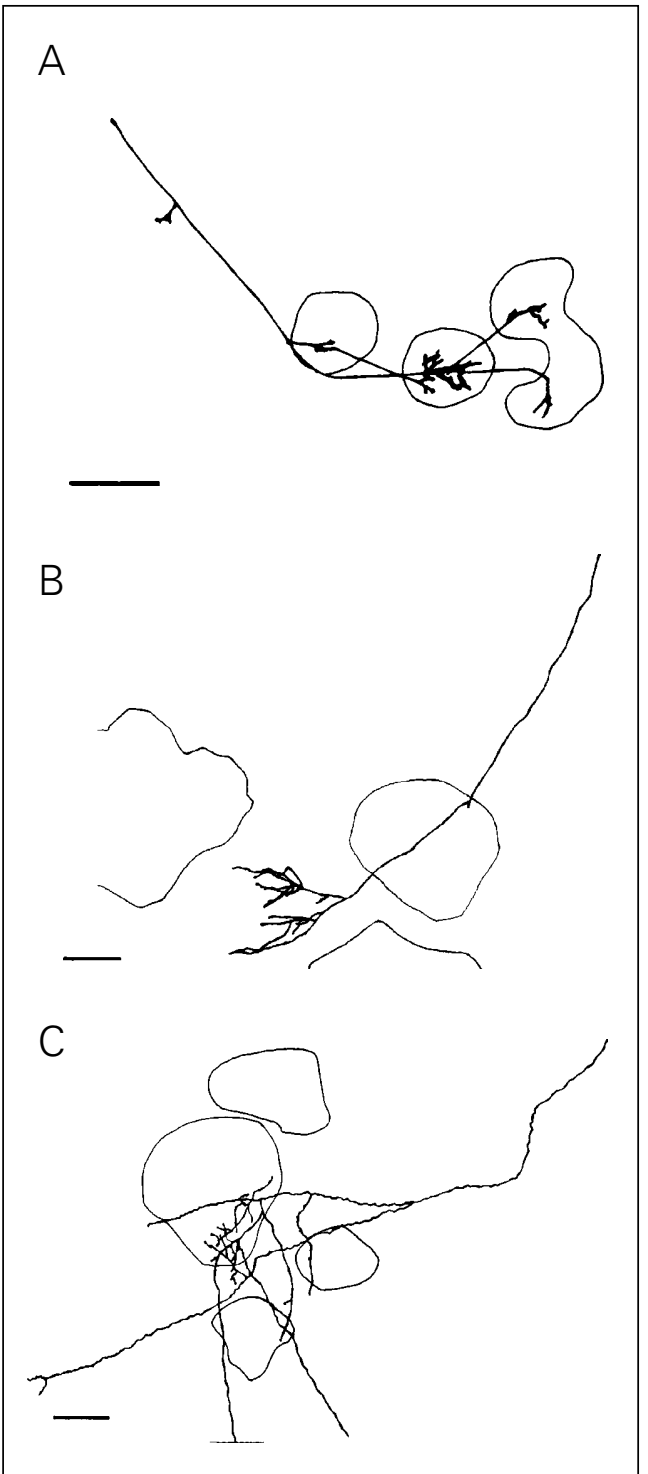

Table 4 - Segregation pattern of intrinsic axon terminals of V1 of Cebus apella.

\begin{tabular}{lll}
\hline Axon terminals & Type & Cortical region \\
\hline $1,2,3$ & la & exclusively inside blobs \\
$4,5,6$ & la & exclusively outside blobs \\
7 & la & mainly but not exclusively outside blobs \\
$8,9,10,11,12$ & la & both inside and outside blobs \\
13,14 & lb & mainly but not exclusively outside blobs \\
15 & Ib & exclusively outside blobs \\
16 & lb & mainly but not exclusively inside blobs \\
17 & lb & both inside and outside blobs
\end{tabular}

tions in relation to the modular organization of V1. In order to correlate intrinsic axon terminals with the cortical modular organization we used the procedures described in the Methods section. Quantitative analysis of the injection sites revealed diameters of 237 to $520 \mu \mathrm{m}$ that in some cases were wider than the blob diameters (Figure 2). Iontophoretic injections produced a dense labeling of fibers around the injection sites, with a radial pattern of distribution similar to that described by Livingstone and Hubel $(13,14)$. Retrograde labeling around the injection site was less intense. Primary segments of axon terminals of type I could be followed to distances varying from 160 to $732 \mu \mathrm{m}$. Their patches, in extreme cases, covered cortical areas measuring $80 \times 390 \mu \mathrm{m}$ in tangential sections. Axon terminals were reconstructed from the borders of the injection sites to the end of their distal processes. Double labeling for biocytin and CYTOX in tangential sections of the operculum permitted an easier definition in a single section of the limits of the blobs relative to the area covered by axon terminals. Only type I axon terminals were used for this analysis and the results are summarized in Table 4.

We found three groups of axon terminals with respect to the degree of modular segregation: 1) complete segregation of terminals inside (Figure 8A) or outside blobs (Figure $8 \mathrm{~B})$, and 2) incomplete segregation of terminals with indiscriminate innervation of both blob and interblob areas (Figure 8C).

\section{Metric features of intrinsic axon terminals}

Thirty axon terminals were analyzed using 3-D automatic microscopy and 14 of them were used for statistical analysis. Four axon terminals from group II and 10 from group I were selected for quantitative analysis based on the criteria previously described. For quantitative analysis the following anatomical parameters were considered: total length of the axonal tree, branching angle 

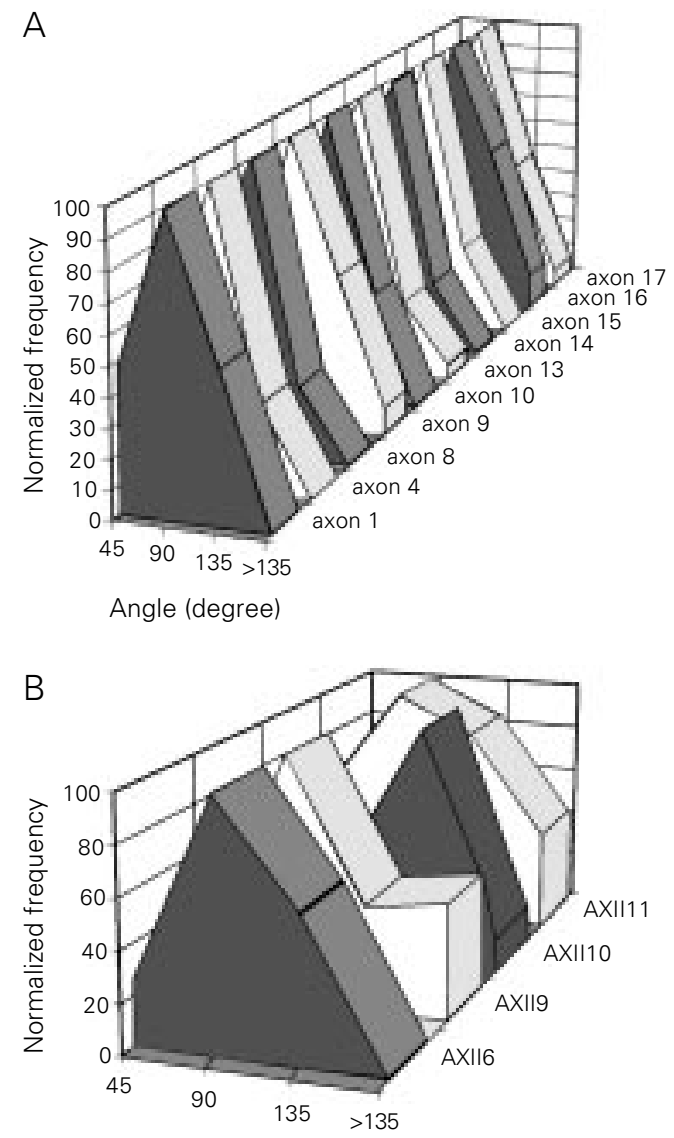

Angle (degree)

Figure 9 - Branching angles of individual axon terminals of V1 of Cebus apella. A, Type I; B, type II.

and densities of branching points, branches, and boutons. Analysis of these parameters revealed striking differences between groups I and II. Figure 9 illustrates the results of branching angle analysis for individual axon terminals of types I (Figure 9A) and II (Figure 9B). Note the sharp distribution of branching angles for type I axons (peak values between $45^{\circ}$ and $90^{\circ}$ ) compared with the distribution for type II which, although displaying a wider distribution, shows a higher frequency of values greater than $90^{\circ}$.

On average, group I tended to show acute branching angles $\left(67.5 \pm 29^{\circ}\right.$, mean $\pm \mathrm{SD}, \mathrm{N}$ $=338$ ) when compared with group II, which displayed straight angles $\left(91.2 \pm 34.3^{\circ}\right.$, mean $\pm \mathrm{SD}, \mathrm{N}=120 ; \mathrm{P}<0.05, t$-test $)$.

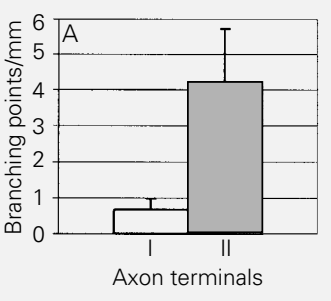

Figure 10 shows that group II axon terminals tended to present, on average, a more ramified and compact branching pattern (4.22 \pm 1.5 branching points $/ \mathrm{mm}$, mean $\pm \mathrm{SD}, \mathrm{N}=$ 134) (Figure 10A), smaller cortical territory innervation with shorter $(5.1-186 \mu \mathrm{m})$ and denser intermediate segments $(8.43 \pm 3.4$ segments/mm, mean $\pm \mathrm{SD}, \mathrm{N}=259$ ) (Figure 9B) but higher density of potential synaptic sites $(21.2 \pm 8.1$ boutons $/ \mathrm{mm}$, mean $\pm \mathrm{SD}, \mathrm{N}$ = 634) (Figure 9C) when compared with group I that showed $0.67 \pm 0.3$ branching points/mm, mean $\pm \mathrm{SD}, \mathrm{N}=341 ; 1.43 \pm 0.7$ segments $/ \mathrm{mm}$, mean $\pm \mathrm{SD}, \mathrm{N}=702$, and 5.7 \pm 3.9 boutons $/ \mathrm{mm}$, mean $\pm \mathrm{SD}, \mathrm{N}=2518$, and innervated a larger cortical area with longer intermediate segments $(5.0-841 \mu \mathrm{m})$. In all cases, the $t$-test for evaluation of statistical differences was significant $(\mathrm{P}<0.05)$.

\section{Discussion}

\section{Cortical lamination}

In the present study we used the terminology of cortical lamination previously published by Lachica et al. (4), and modified by Hässler (22). Two aspects of our results made the use of this nomenclature more adequate: 1) supragranular layers in V1 of primate visual cortex give rise to both extrinsic and intrinsic projections (12). Thus, layer IVb of Brodmann's terminology which has been labeled in Cebus apella, both intrinsically (present study) and by implanted saturated pellets of biocytin in V2 (Amorim AKJ and PicançoDiniz CW, unpublished results), can be included as part of layer III. 2) Large intrinsically and extrinsically labeled pyramidal cells

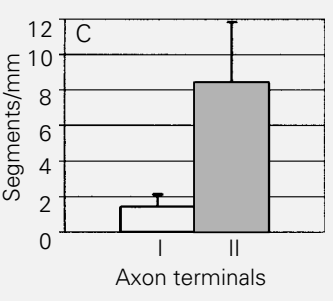

Figure 10 - Metric features of intrinsic axon terminals of $\mathrm{V} 1$ comparing density values per $\mathrm{mm}$ of axon length. $A$, Branching points; $B$, boutons; $C$, segments. 
have been found in layer IVb (Brodmann's terminology) of Cebus in the present study, an uncommon finding for layer IV of the primate brain, as reported by Casagrande and Kaas (12).

The present results confirm and extend previous data demonstrating that biocytin can be employed for high resolution retrograde and anterograde neurotracing $(7,18,25-29)$ combined with cytochrome oxidase (26) and with NADPH-diaphorase with better results than those obtained with peroxidase in primate visual cortex (11).

Anatomical reconstruction of dendritic trees or axon terminals in single thick sections assumes that part of the tree is lost in adjacent sections. Incomplete reconstructions require the use of only metric features that do not depend on the order of the segment for data comparison. This measure leads to both qualitative and quantitative consistency of the data, which show, on average, the same tendency in each individual group. The quantitative differences observed between groups I and II were statistically significant, a fact that validates the choice of the analytical approach.

We cannot assume that all labeled fibers represent intrinsic axon terminals of V1 in $100 \%$ of the cases, inasmuch as there exists the possibility of labeling passing fibers. However, in most cases reported in previous studies using biocytin as neurotracer $(7,21,25,26$, $28,29)$ the degree of labeling of passing fibers was related to the amount of tracer delivered as well as to the extent of the tissue damage. Iontophoretic injections such as those used in the present study produce minimal damage and small injection sites (100-200 $\mu \mathrm{m})$. However, it is important to consider that Chevalier et al. (30) disagree with this argument and report passing fibers labeled after iontophoretic injections, thereby reopening the question.

\section{Cell morphology and laminar distribution}

The present paper described retrogradely labeled cells with different morphological characteristics and laminar distribution. We found pyramids, star pyramids and stellate cells in layers II, IIIa (III), IIIb $\alpha$ (IIIb), IIIb $\beta$ (IVa) and IIIc (IVb). These results are similar to those reported by Rockland and Lund (16) and Yoshioka et al. (8) who used HRP pressure or biocytin iontophoretic injections, respectively. However, one of the most intriguing questions that remains open is the complete absence of labeling of dendritic arbors of non-pyramidal local neurons that nonetheless had their axon terminals labeled (type II axon terminals).

Using intracellular HRP injections Anderson et al. (6) have found similar spine cells participating in intrinsic projections of $\mathrm{V} 1$ in Macaca nemestrina. In agreement with Rockland and Lund (16), we found a number of clusters of pyramidal and stellate cells in the supragranular layers of $\mathrm{V} 1$ of Cebus apella that deserve further investigation from a functional point of view. Similar to the results of Blasdel et al. (31), these projections were asymmetrical in relation to the injection site, with strong labeling on one side as compared to the other. In cases of iontophoretic injections located in layers II and III the infragranular labeled cells were found in layer V, similar to the results reported by Livingstone and Hubel (14). These labeled cells also appear in layer VI when the injection site includes layer IV in addition to layers II and III. This result may possibly indicate interlaminar axonal projections from neurons of layers II and III or, as suggested by others $(4,8,32)$, selective uptake by damaged axons or dendrites of infragranular pyramidal cells passing through the granular or supragranular layers, in register with the injection site.

An interesting finding was the similarity observed between intrinsic elements of V1 and V2 in Cebus apella as regards cell types and laminar distribution (Amorim AKJ and Picanço-Diniz CW, unpublished results). This result could be interpreted in two possible ways: either as evidence that the elements that 
project intrinsically to $\mathrm{V} 1$ also project to $\mathrm{V} 2$ by means of collateral axonal branching, or that the fibers which are crossing V1 to reach their targets in V2 have been labeled as a consequence of damage. In the latter case the possibility of uptake of biocytin by passing fibers remains open. Against this possibility is the fact that similar patterns of labeling are obtained irrespective of the amount of tissue damaged $(8,16,31$, and the present results). The first hypothesis, i.e., the same cells projecting both intrinsically and extrinsically, is supported by the results obtained after isolated injections into layers II and III or IVb (31) which show retrogradely labeled pyramidal cells and axon terminals forming patches in layers II and III. In the case of layer IVb injections these authors found pyramids, spiny stellate cells and axon terminals in the same layer. These data reinforce the results of intracellular injections by Anderson et al. (6) that revealed pyramidal, star pyramidal and stellate cells projecting both intrinsically and extrinsically.

\section{Axon terminals}

The morphological details of the axon terminals of groups I and II in V1 of Cebus monkey are similar to those previously described by Martin and Whitteridge (33) for the striate cortex of the cat. These authors correlated these two axonal types with excitatory and inhibitory functions, respectively. In addition, the fact that we found a higher proportion of type I axons may reflect the proportion of excitatory neurons $v s$ inhibitory ones described. The use of different metric features in the analysis of the same group may suggest the existence of subgroups, as pointed out by a qualitative analysis of group I. However, we have not yet perfomed significant sampling analyses to support this hypothesis, which remains open for future investigation. Whether or not there are subgroups of axon terminals in group I, they tend to innervate a larger extent of cortical territory and to dis- play fewer potential synaptic sites (bouton densities/mm) when compared to group II. This hypothetically lower synaptic density could be compensated for by the higher frequency of occurrence of terminals of group I than of group II. We propose that spine neurons are related to group I and smooth neurons to group II $(34,35)$. A comparison of the present results in terms of pattern of arborization, size and morphology of boutons for axon terminals of type II with those reported by Lund (36) and Lund and Yoshioka (37) permits us to correlate these terminals with smooth neurons of inhibitory local circuits. Despite the fact that there are insufficient data to exclude the participation of smooth cells in long distance horizontal connections (38), intracellular studies (39) imply the presence of basket cells in intrinsic connections covering 1.0-1.5 mm of the cortex, and suggest that these cells show target specificity, making synaptic contacts with dendritic spines of pyramidal cells. Thus, by restricting themselves to smaller cortical territories they would increase their densities per cortical volume, thereby compensating for their numerical inferiority. The quantitative data of the present study seem to support this hypothesis.

The most interesting aspect of the quantitative analysis of the axon terminals is the fact that it provides additional support to the major morphological subdivision of neocortical neurons into two simple groups based on the presence or absence of spines in their dendrites. These data, together with the fact that there are only two types of synapses described (gray types I and II), may be useful for identifying similarities rather than diversities of anatomical characteristics (39). Further investigation is necessary, using electrophysiological intracellular labeling, immunocytochemistry and neuropharmacology approaches, to obtain essential information about the morphofunctional properties of $\mathrm{V} 1$ neurons of Cebus monkey that give rise to axon terminals of groups I and II. 


\section{Modular and laminar distribution of axon terminals}

A preliminary question to be considered here is the difficulty in defining the limits of blobs $(4,8,16,40)$. In order to reduce errors we have taken some precautions: 1 ) we used low power objectives to reconstruct the borders of the blobs because they permit a better visualization of their limits. 2) For the reconstruction of the terminals we used a high power objective which rendered impossible simultaneous visualization of the blobs. 3) These reconstructions were recorded by using the $\mathrm{x}, \mathrm{y}$ and $\mathrm{z}$ coordinates fed automatically to a computer system, thus removing interference from the researcher. Taking all of this into consideration, it was possible to observe irregularities in the shape and spatial distribution of the blobs that contradict with homogeneous aspects described in the literature $(1,13,14)$. This variability agrees with the results reported by others $(7,8,16,40)$. It is important to emphasize, however, that on some occasions it was very difficult to delineate the limits of the blobs and to distinguish them from the background.

A particular type of dense and very small blobs observed in the vicinity of blood ves- sels, as previously described by Franca (41) forSaimiri sciureus, was not considered here as part of the modular organization of V1 and was not taken into consideration for the purposes of the present analysis. The different histochemical procedures, neurotracers and species used, as well as different injection sizes, partially explain the conflicting results in the literature. However, it is interesting to note that none of these factors could explain the existence of individual axon terminals innervating both blobs and interblob regions. This finding may indeed suggest the presence of anatomical circuits that link different modules of $\mathrm{V} 1$, a result that is in close agreement with recent findings of Lachica and Casagrande (42) who demonstrated that the parallel channels from the lateral geniculate nucleus are not completely segregated in these modules (blobs of layers IIIb $\alpha$ and IIIb $\beta$ (IIIb and IVa) and that they receive direct projections from both layers IV $\alpha$ (IVc $\alpha$ ) and IV $\beta$ (IVc $\beta)$. There is evidence for both incomplete $(3,4,7,43)$ and complete $(5,14,15)$ segregation of the flow information in $\mathrm{V} 1$ of the primate brain. These data gain further support from functional results that revealed interconnections between columns with similar and different orientation (44-47).

\section{References}

1. McGuire BA, Gilbert CD, Rivlin PK \& Wiesel TN (1991). Targets of horizontal connections in macaque primary visual cortex. Journal of Comparative Neurology, 305: 370-392.

2. Lachica EA, Beck PD \& Casagrande VA (1992). Parallel pathways in macaque monkey striate cortex: anatomically defined columns in layer III. Proceedings of the National Academy of Sciences, USA, 89: 3566-3570.

3. Yoshioka T, Blasdel GG, Levitt JB \& Lund JS (1992). Patterns of lateral connections in macaque visual area $\mathrm{V} 1$ revealed by biocytin histochemistry and functional imaging. Society for Neuroscience Abstracts, 18: 299
4. Lachica EA, Beck PD \& Casagrande VA (1993). Intrinsic connections of layer III of striate cortex in squirrel monkey and bush baby: correlations with patterns of cytochrome oxidase. Journal of Comparative Neurology, 329: 163-187.

5. Amir Y, Harel M \& Malach R (1993). Cortical hierarchy reflected in the organization of intrinsic connections in macaque monkey visual cortex. Journal of Comparative Neurology, 334: 19-46.

6. Anderson JC, Martin KA \& Whitteridge D (1993). Form, function and intracortical projections of neurons in the striate cortex of the monkey Macacus nemestrinus. Cerebral Cortex, 3: 412-420.

7. Lund JS, Yoshioka T \& Levitt JB (1993). Comparison of intrinsic connectivity in different areas of macaque monkey cerebral cortex. Cerebral Cortex, 3: 148-162.
8. Yoshioka T, Levitt JB \& Lund JS (1994). Independence and merger of thalamocortical channels within macaque monkey primary visual cortex: anatomy of interlaminar projections. Visual Neuroscience, 11: 467-489.

9. Callaway EM \& Wiser AK (1996). Contributions of individual layer 2-5 spiny neurons to local circuits in macaque primary visual cortex. Visual Neuroscience, 13: 907-922.

10. Yoshioka T, Blasdel GG, Levitt JB \& Lund JS (1996). Relation between patterns of intrinsic lateral connectivity, ocular dominance, and cytochrome oxidase-reactive regions in macaque monkey striate cortex. Cerebral Cortex, 6: 297-310. 
11. Lund JS, Yoshioka T \& Levitt JB (1994). Substrates for interlaminar connections in area $\mathrm{V} 1$ of macaque monkey cerebral cortex. In: Peters A \& Rockland KS (Editors), Cerebral Cortex. Vol. 10. Plenum Press, New York.

12. Casagrande VA \& Kaas JH (1994). The afferent, intrinsic, and efferent connections of primary visual cortex in primates. In: Peters A \& Rockland KS (Editors), Cerebral Cortex. Vol. 10. Plenum Press, New York.

13. Livingstone MS \& Hubel DH (1984). Anatomy and physiology of a color system in the primate visual cortex. Journal of Neuroscience, 4: 309-356.

14. Livingstone MS \& Hubel DH (1984). Specificity of intrinsic connections in primate primary visual cortex. Journal of Neuroscience, 4: 2830-2835.

15. Malach R (1991). Relationship of biocytin labeled neuronal processes to the cytochrome oxidase (CO) rich blobs in monkey striate cortex. Society for Neuroscience Abstracts, 17: 117.

16. Rockland KS \& Lund JS (1983). Intrinsic laminar lattice connections in primate visual cortex. Journal of Comparative Neurology, 216: 303-318.

17. Amorim AKJ \& Picanço-Diniz CW (1996). Morphometric analysis of intrinsic axon terminals of Cebus monkey area 17. Brazilian Journal of Medical and Biological Research, 29: 1363-1368.

18. Picanço-Diniz CW, Silveira LCL, Yamada ES \& Martin KAC (1992). Biocytin as a retrograde tracer in the mammalian visual system. Brazilian Journal of Medical and Biological Research, 25: 57-62.

19. Wong-Riley MTT (1979). Changes in the visual system of monocularly sutured or enucleated cats demonstrable with cytochrome oxidase histochemistry. Brain Research, 171: 11-28.

20. Scherer-Singler $U$, Vincent $S R$, Kimura $H$ \& McGeer EG (1983). Demonstration of a unique population of neurons with NADPH-diaphorase histochemistry. Journal of Neuroscience Methods, 9: 229-234.

21. Shu S, Ju G \& Fan L (1988). The glucose oxidase-DAB-nickel method in peroxidase histochemistry of the nervous system. Neuroscience Letters, 85: 169-171.

22. Hässler R (1967). Comparative Anatomy of Central Visual Systems in Day- and Night-Active Primates. Plenum Press, New York.

23. Brodmann K (1909). Vergleichende Lokalisationslehre der Grosshimrinde. Barth, Leipzig.
24. Rosa MGP, Gattass $R$ \& Soares JGM (1991). A quantitative analysis of cytochrome oxidase-rich patches in the primary visual cortex of Cebus monkeys: topographic distribution and effects of late monocular enucleation. Experimental Brain Research, 84: 195-209.

25. King MA, Louis PM, Hunter BE \& Walker DW (1989). Biocytin: a versatile anterograde neuroanatomical tract-tracing alternative. Brain Research, 497: 361-367.

26. Lachica EA, Mavity-Hudson JA \& Casagrande VA (1991). Morphological details of primate axons and dendrites revealed by extracellular injection of biocytin: an economic and reliable alternative to PHA-L. Brain Research, 564: 1-11.

27. Kenan-Vaknin G, Katz $H$ \& Malach $R$ (1992). Golgi-like staining of visual cortex cells obtained by extracellular biocytin application in vitro. Brain Research, 571: 338-341.

28. McDonald AJ (1992). Neuroanatomical labeling with biocytin: a review. Neuroreport, 3: 821-827.

29. Sorensen JC, Tonder N \& Zimmer J (1993). Biocytin pellets: an alternative technique for massive anterograde labeling of neuronal pathways in vivo and in vitro. Brain Research, 608: 338-344.

30. Chevalier G, Deniau JM \& Menetrey A (1992). Evidence that biocytin is taken up by axons. Neuroscience Letters, 140: 197199.

31. Blasdel GG, Lund JS \& Fitzpatrick D (1985). Intrinsic connections of macaque striate cortex: axonal projections of cells outside lamina 4c. Journal of Neuroscience, 5: 3350-3369.

32. Fitzpatrick D, Lund JS \& Blasdel GG (1985). Intrinsic connections of macaque striate cortex: afferent and efferent connections of lamina 4c. Journal of Neuroscience, 5: 3329-3349.

33. Martin KAC \& Whitteridge D (1984). Form, function and intracortical projections of spiny neurons in the striate visual cortex of the cat. Journal of Physiology, 353: 463-504.

34. Beaulieu C, Kisvarday Z, Somogyi P, Cynader M \& Cowey A (1992). Quantitative distribution of GABA-immunopositive and -immunonegative neurons and synapses in the monkey striate cortex (area 17). Cerebral Cortex, 2: 295-309.

35. Fitzpatrick D, Lund JS, Schmechel DE \& Towles AC (1987). Distribution of GABAergic neurons and axon terminals in the macaque striate cortex. Journal of Comparative Neurology, 264: 73-91.
36. Lund JS (1987). Local circuit neurons of macaque monkey striate cortex: I. Neurons of laminae $4 \mathrm{c}$ and $5 \mathrm{a}$. Journal of Comparative Neurology, 257: 60-92.

37. Lund JS \& Yoshioka T (1991). Local circuit neurons of macaque monkey striate cortex: III. Neurons of laminae 4b, 4a, 3b. Journal of Comparative Neurology, 311: 234-258.

38. Lund JS (1988). Anatomical organization of macaque monkey striate visual cortex. Annual Review of Neuroscience, 11: 253288.

39. Douglas RJ \& Martin KAC (1991). Neocortex. Oxford University Press, New York.

40. Horton JC (1984). Cytochrome oxidase patches: a new cytoarchitectonic feature of monkey visual cortex. Philosophical Transactions of the Royal Society of London, Series B, 304: 199-253.

41. Franca JG (1993). NADPH-diaforase no córtex visual do macaco-de-cheiro (Saimiri sciureus): Atividade histoquímica nas áreas 17 e 18. Master's thesis, Instituto de Biofísica Carlos Chagas Filho, Universidade Federal do Rio de Janeiro, Rio de Janeiro.

42. Lachica EA \& Casagrande VA (1993). The morphology of collicular and retinal axons ending on small relay ( $w$-like) cells of the primate lateral geniculate nucleus. Visual Neuroscience, 10: 403-418.

43. Leventhal AG, Thompson KG, Liu D, Zhou Y \& Ault ST (1995). Concomitant sensitivity to orientation, direction, and color of cells in layers 2,3 , and 4 of monkey striate cortex. Journal of Neuroscience, 15: 1808-1818.

44. Hübener M \& Bolz J (1992). Relationships between dendritic morphology and cytochrome oxidase compartments in monkey striate cortex. Journal of Comparative Neurology, 324: 67-80.

45. Malach R (1992). Dendritic sampling across processing streams in monkey striate cortex. Journal of Comparative Neurology, 315: 303-312.

46. Malach $R$, Amir $Y$, Harel $M$ \& Grinvald $A$ (1993). Relationship between intrinsic connections and functional architecture revealed by optical imaging and in vivo targeted biocytin injections in primate striate cortex. Proceedings of the National Academy of Sciences, USA, 90: 1046910473.

47. Malach R (1994). Cortical columns as devices for maximizing neuronal diversity. Trends in Neurosciences, 17: 101-104. 